\title{
Does the Use of Hair Dyes Increase the Risk of Developing Breast Cancer? A Meta-analysis and Review of the Literature
}

\author{
RITIKA GERA $^{1,2}$, RAMIA MOKBEL ${ }^{3}$, IVANNA IGOR ${ }^{1}$ and KEFAH MOKBEL ${ }^{1}$ \\ ${ }^{1}$ The London Breast Institute, Princess Grace Hospital, HCA Healthcare, London, U.K.; \\ ${ }^{2}$ University College London Medical School, London, U.K.; \\ ${ }^{3}$ Department of Dermatology, St. Mary's Hospital and Imperial College Healthcare, London, U.K.
}

\begin{abstract}
Background/Aim: Hair dye may contain mutagenic compounds which could be associated with an increased incidence of breast cancer in women who use it. The aim of this study was to examine the association between the personal use of hair dyes and the risk of breast cancer. Materials and Methods: We conducted a literature review of epidemiological studies reporting breast cancer-specific risks among hair dye users versus non-users. The data for the incidence of breast cancer following the 'ever' use of hair dye in studies which met the inclusion criteria was analysed using a meta-analysis. The relative risk ratio $(R R)$ and $95 \%$ confidence intervals (CI) were determined. Results: A total of eight case-control studies published between 1980 and 2017 met the selection criteria and were included in the meta-analysis. Compared to non-users, using a random effects model and the Duval and Tweedie's trim and fill procedure to adjust for publication bias in the presence of between studies heterogeneity, the adjusted RR for women using hair dyes was 1.1885 (95\% CI=1.03228-1.36835). This indicates an $18.8 \%$ increased risk of future development of breast cancer among hair dye users. Conclusion: Although further work is required to confirm our results and clarify potential mechanisms, our findings suggest that exposure to hair dyes may contribute to an increased breast cancer risk.
\end{abstract}

Hair dye is a commercial product that many members of the public, specifically women, use to cosmetically alter their appearance for a variety of reasons, from covering up grey hair to completely changing the appearance of their hair. Within Europe and the USA, it has been estimated that more

Correspondence to: Kefah Mokbel, London Breast Institute, Princess Grace Hospital, London, U.K. Tel: +44 2079082040, e-mail: kefahmokbel@hotmail.com

Key Words: Hair dye, breast cancer, meta-analysis, literature review. than one-third of women over the age of eighteen and approximately $10 \%$ of men over the age of 40 use some type of hair dye (1). Although certain components of hair dye have been identified as harmful to human health based on laboratory and animal studies and have consequently been banned by the European Commission (2), hair dyes continue to contain chemicals which are potentially carcinogenic.

Most permanent hair dye products contain aromatic amines such as para-phenylenediamine (PPD: $\mathrm{C}_{6} \mathrm{H}_{8} \mathrm{~N}_{2}$ ), a well-documented potent skin sensitizer, as primary intermediates. They are mixed with couplers and an oxidizing agent such as hydrogen peroxide and the oxidized products impart hair coloration. Elevated levels of DNAadducts of aromatic amines have been detected in breast epithelial cells of hair dye users. Furthermore in vitro genotoxicity studies showed PPD to be mutagenic and in vivo animal studies demonstrated that topical and subcutaneous administration was associated with an increased incidence of benign and malignant mammary tumours (3). The International Agency for Research on Cancer (IARC) has classified such aromatic amines as probably carcinogenic to humans who are exposed to them on an occupational basis such as hairdressers and barbers (2A risk category) but not to humans who use them on a personal basis (4). It should be noted that the epidemiological studies in this meta-analysis had no specific focus on examining any potential existing causative relationship between the use of PPD in hair dye and the subsequent incidence of breast cancer, so it is not possible to comment specifically on this. PPD has been included as an example of a chemical widely used in hair dye which merits further detailed epidemiological investigation due to its probable carcinogenicity.

Epidemiological studies investigating the relationship between the use of hair dyes and breast cancer risk have yielded conflicting results. Therefore, from a public health perspective, it is important to examine the various epidemiological studies which investigated this relationship 
and subsequently perform a meta-analysis to improve statistical power and attempt to determine if there is any overall increased risk.

\section{Materials and Methods}

Literature search. The initial searches were conducted on online databases: PubMed, Science Direct, NCBI. Search terms used were: 'hair dye' and 'breast cancer'. Abstracts were found and then scrutinised. An abstract which showed clear evidence of not fulfilling the inclusion criteria was rejected. If the abstract did not conflict with the inclusion criteria the full document was sourced and examined. In the instance that the full document fit the inclusion criteria, the study was included in the meta-analysis and this process was repeated.

Inclusion and exclusion criteria. The epidemiological studies included in this meta-analysis must have had female participants with no previous diagnosis of breast cancer. The studies must have reported a RR/odds ratio (OR) and a $95 \%$ confidence interval and included data concerning the 'ever' versus 'never' use of hair dye. The studies must have also been found as full text journal articles along with the abstract; studies were excluded if only the abstract was found. The studies must have reported full selection criteria for cases and controls and provided the baseline category information.

Studies examining any non-breast related cancer as a case were excluded. Any studies which did not make hair dye a key risk factor being investigated or in which the key hair product investigated was not hair dye were excluded. Any studies which did not publish the full raw data collected or include adequate controls were also excluded.

Any epidemiological studies which failed to meet the above inclusion criteria/met any of the above exclusion criteria were excluded.

The search within NCBI yielded 47 scientific and medical abstracts/citations within PubMed, 1997 full-text journal articles in PubMed Central and 3257 search results in Science Direct. Of these, 24 studies were considered relevant and initially selected to examine further of which 8 studies were included in this meta-analysis (5-6, $8-10,12-14)$ and 16 were excluded $(10,15-29)$. The studies fulfilling the selection criteria were published between 1980 and 2017 and contained a total of 38037 participants.

Analysis. The following data was recorded from the studies: RR/OR, lower and upper limits of CI, number of cancer and control cases, and country of study. To perform the meta-analysis, the RR and CIs were used as a measurement of the effect of 'ever' use of hair dye on the incidence of breast cancer.

The meta-analysis was conducted using models of both fixed and random effects. Statistical heterogeneity was tested for using the following: the funnel plot, $\mathrm{I}^{2}$, and Tau squared. Numbers in the range $0-100 \%$ were used as an indication of statistical heterogeneity. In cases where the $p$-value was less than 0.1 , homogeneity could not be assumed. Estimates were taken from the random effects model

The risk for publication bias was calculated using the Begg and Mazumdar rank correlation. Egger's regression intercept was also used. Duval and Tweedie's trim and fill was used to adjust for publication bias within the meta-analysis. To describe the results of the meta-analysis, a forest plot was created.

\section{Results}

Using a random-effects model the pooled RR was estimated to be $1.1465(95 \% \quad \mathrm{CI}=0.9962-1.3194)$. The $p$-value calculated from the z-test is 0.056 , which indicates significance at the $6 \%$ significance level. The probability that the true RR is less than 1 is $2.84 \%$ (Figure 1 ).

To investigate the risk of publication bias and statistical heterogeneity, a funnel plot (Figure 2) was created. The funnel plot is fairly symmetrical which suggests a reasonable degree of statistical heterogeneity. This is supported by the result of the 'I' squared test: 73.89432 . However, the tau squared test seemed to indicate considerable statistical heterogeneity: 2.70 .

There is one outlier within the funnel plot (Figures 2 and 3 ). The funnel plot does not give an impression of severe publication bias. However, there are too few studies to make a reasonable inference from the plot. Duval and Tweedie's trim and fill found no necessity to trim studies to the left of the combined effect, but to the right of it, one study was determined. The adjusted combined effect according to the Duval and Tweedie's Trim and Fill procedure revealed a RR of $1.1885(95 \% \mathrm{CI}=1.03228-1.36835)$. The lower bound of the confidence interval clearly exceeds 1 .

Using a fixed effects model revealed similar estimates of the RR being $1.16548(95 \% \mathrm{CI}=1.098-1.237)$ prior to adjustment and $1.14680(95 \% \quad \mathrm{CI}=1.079-1.219)$ after adjustment for publication bias in the presence of between studies heterogeneity.

After examining the excluded studies, an unweighted RR was calculated by averaging the OR/RR/hazard ratio (HR) present in each suitable individual excluded study. The studies $(n=6)$ included in the average $(16-17,20,23,27-28)$ fulfilled the following basic criteria: they should examine only women, the total number of participants in the study should be available and they should provide the RR, OR, or HR of developing breast cancer following the ever use of hair dye. There was a total of 317,963 women included in this average. The average unweighted RR was 2.295 , with the range of all 6 excluded studies included in this calculation being 0.93-8.62. The average OR weighted according to the total sample size of the study was 1.051 . The study with the highest RR of 8.62 (20) also had one of the smallest sample sizes: 200 women in total. This study was excluded from the meta-analysis due to its many limitations which include: the seemingly anomalous result and the very small sample size. It is not possible to comment on the validity of this average because they were excluded from this meta-analysis and so were not examined to the fullest degree.

Five studies excluded in this meta-analysis $(21,23,27-$ 29) were prospective. Of these, three studies $(23,27-28)$ were included in the average of the excluded studies 


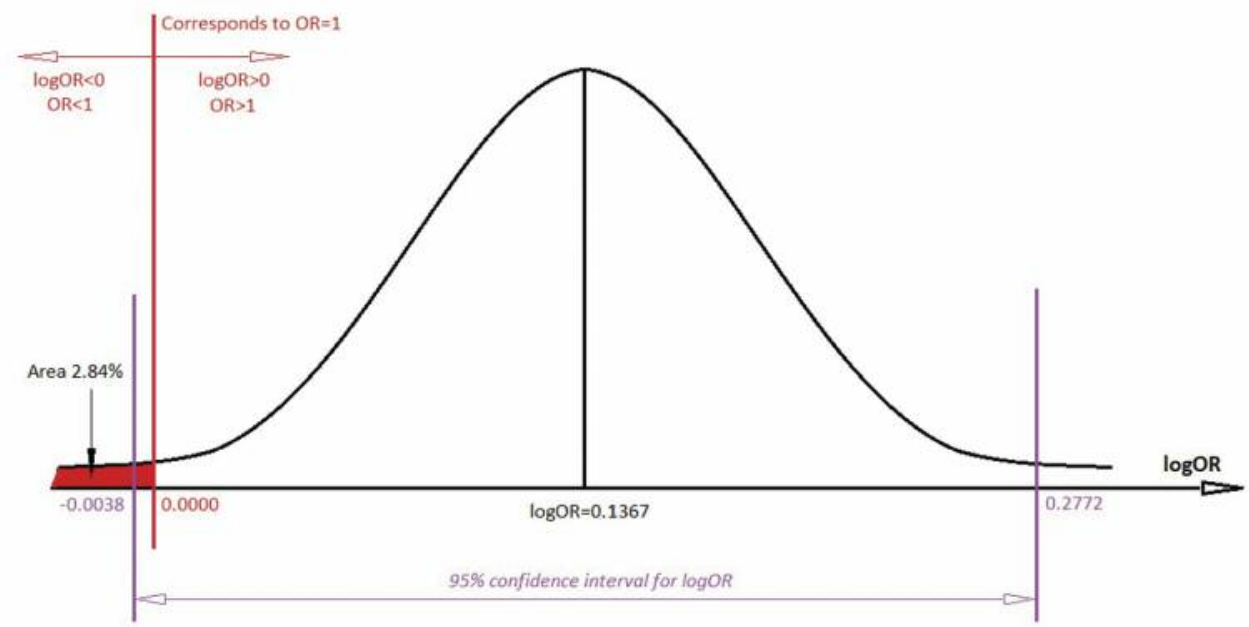

Figure 1. The normal distribution of log odds ratio demonstrating that the probability of the combined odds ratio being less than 1 is only 2.84 percent.

calculated above. These prospective studies were excluded from the meta-analysis for various reasons: the death rate was recorded instead of the rate of incidence of breast cancer $(21,29)$, there was no information provided on the number of controls used (23), an HR was used instead of an OR/RR and the study had a high focus on other types of cancer (27), and there was no baseline category information published (28). Due to the large number of excluded prospective studies, the reliability of this statistical analyses has decreased. There was no significant correlation between the use of hair dye and incidence of/death from breast cancer in all five prospective studies.

\section{Discussion}

The findings of this meta-analysis suggest that the personal use of hair dyes is associated with an increased risk of developing breast cancer. Its strength lies in providing a combined estimate of several case-control studies which reported raw data and met strict inclusion criteria. However, the lack of accurate information regarding different exposure characteristics across multiple studies included in this metaanalysis prohibited the analysis of duration and frequency of exposure and type of hair dye used in the context of breast cancer risk. Therefore, our study does not provide any insights into the dose-effect relationship or the chemical constituents implicated in potential causation. There was significant heterogeneity among studies and no uniform adjustment for confounding factors. It is also important to note that there can be a vast amount of variation between different populations, from genetics to cultural habits surrounding hair dye application to socioeconomic status.
There were eight epidemiological studies included in this meta-analysis and each study has been analysed below for its individual strengths and weaknesses. However, a significant number of studies was excluded due to our selection criteria thus limiting the combined sample size and statistical power. Furthermore, the retrospective nature of these studies introduces a significant risk of recall or interviewer bias thus limiting their internal validity. The necessary criteria for future studies examining the relationship between breast cancer risk and hair dye have been outlined later in this article.

Study 1: Relationship of hair dye use, benign breast disease and breast cancer (5). In this retrospective case-control study, female patients between the ages of 20-84 were selected from 3 upstate New York counties to be used as cases and controls. There were 118 cases and 233 controls. The patient list was "stratified by age and county". However, it is unclear to what degree participants were matched up according to age; the maximum difference in age between the case and the control was never specified. If the case was deceased, the interview was conducted with a close member of their family, which greatly increased the risk of recall bias. The number of hair dye applications required to constitute the 'ever' use of hair dye was not strictly quantified at any point. Data was collected through a standardised interviewer, and all interviews were conducted through telephone without any visual prompts which greatly increases recall bias.

The following known risk factors for developing breast cancer were tested for in relation to hair dye exposure prospectively: "previous benign breast disease (BBD), "ever" 


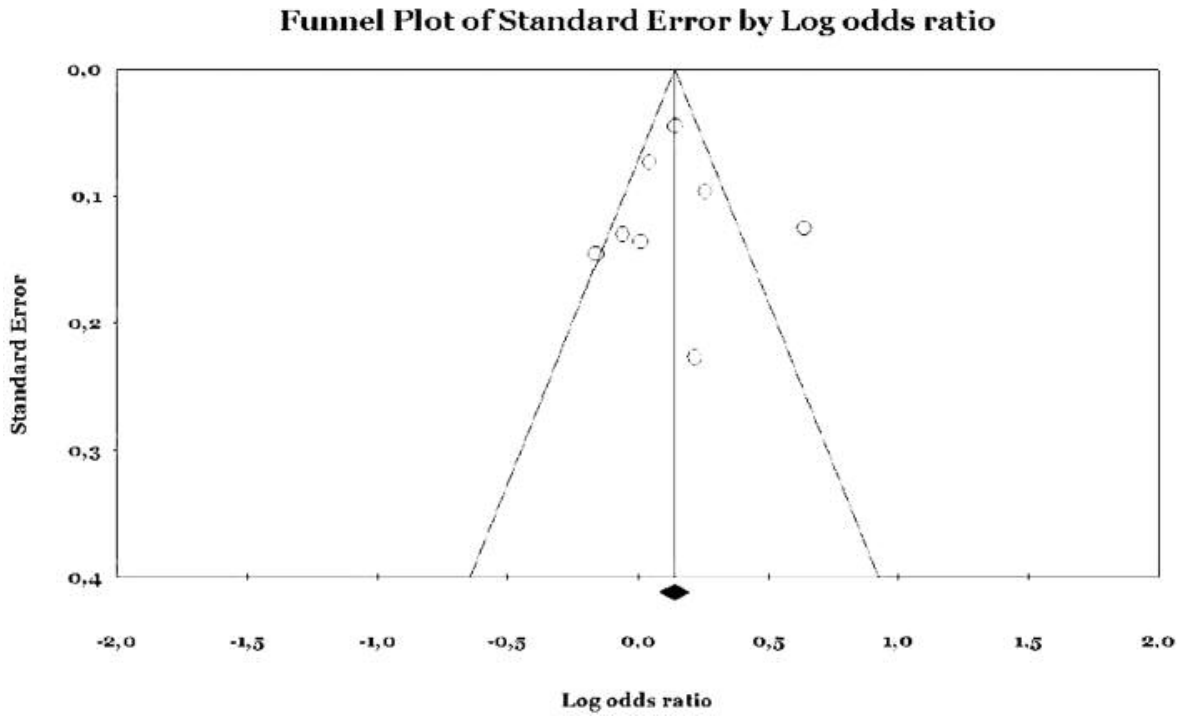

Figure 2. The funnel plot of the studies included in the metanalysis demonstrating heterogeneity and a limited publication bias.

versus "never" pregnant, age at first pregnancy, menopause induced by operation, age at menarche, and education". A multivariate analysis was used to determine the impact of these confounding factors and it found that BBD, both ovaries being removed surgically (surgical menopause) and years of education had a "significant impact on the risk of breast cancer". All three were noted as potential confounding factors and controlled for in "later" experiments. It should also be noted that the BBD was never medically confirmed and was instead based only on cases' answers to the interviewer.

Patients with BBD were found to be significantly more susceptible to any ill effects present in hair dye. A woman with BBD who uses hair dye had a RR of 9.31 for developing breast cancer whereas a woman with BBD who does not use hair dye had a RR of 2.45 ( $p=0.047)$. However, this was based on a relatively small selection of 24 women, which is too few a number to determine a correlation between BBD, use of hair dye and breast cancer.

There were some faults found with the data provided by the investigators. The OR formula is applied to what they term the RR. The formulae applied to calculate the $95 \%$ confidence interval of their RR are not clarified. We have calculated the RR for developing breast cancer following exposure to hair dye as being $1.15(95 \% \mathrm{CI}=0.86-1.55)$.

Study 2: Use of hair colouring products and breast cancer risk: a case-control study in Connecticut (6). This is a casecontrol study conducted on women in Connecticut between the ages of 30-80. Six hundred and eight cases and 609 controls were used. Although there is a large age range, an "effort" was made to try and match the cases and controls within a span of five years. As cases tended to be slightly older than controls, age was a controlled factor in all following analyses. All cases and controls did not have any previous history of cancer, aside from non-melanoma skin cancer. According to a study conducted by the Women's Health Initiative (7), the risk of developing breast cancer following NMSC is $1.07(95 \% \mathrm{CI}=0.95-1.20)$ in postmenopausal women (this analysis used 70246 White and Hispanic women between the ages of 50-79).

Women were selected if they had had surgery related to the breasts at the Yale-New Haven Hospital or if they were residents of Tolland County "between 1st January 1994 and 31st December 1997". "Surgery related to the breasts" is not limited to breast cancer surgery; controls were also chosen by looking at patients undergoing operations on the breasts who were histologically confirmed to not have breast cancer. However, no description of the type of surgery they were having is provided. It is not possible to determine if the risk of developing breast cancer has changed for these patients compared to a female population which has not undergone breast-related surgery, or if these risks differ depending on the type of breast surgery.

At the time of interview, cases and controls had to have been alive. All interviews were conducted in person with the interviewers trained in showing participants the labels of hair products prompt memory, which decreased the chance of recall bias. A "standardised questionnaire" is used to uncover various social, demographic and pathological data, including potential 


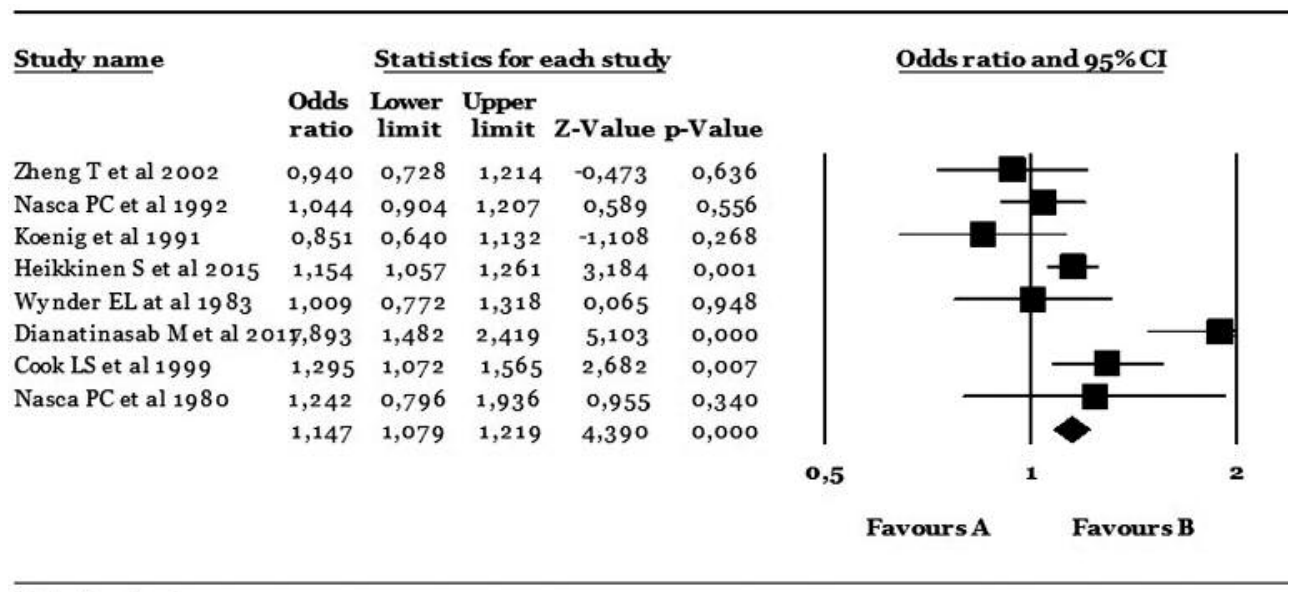

Meta Analysis

Figure 3. A graphical display of estimated results from the eight studies included in the meta-analysis using the Forest plot.

risk factors for developing breast cancer such as family history. Risk was assessed by type and colour of hair dye.

The OR for the 'ever' use of hair dye being linked to the development of breast cancer was 0.9 with a $95 \%$ confidence interval of $0.7-1.2$.

Study 3: An epidemiologic case-control study of breast cancer and exposure to hair dyes (8). This retrospective case-control study was conducted in " 18 contiguous counties of New York State" using women between the ages of 20 and 79 who resided within the study area. Cases were matched to controls by stratifying through the following criteria: sex, birth year, county of residence. 1,617 cases and 1,617 controls were used. Interviews were conducted over the telephone.

Patients who were diagnosed in another hospital outside the study area were identified through the New York State Cancer Registry. This is more effective than focusing on a specific hospital because it accounts and controls for the various confounding variables that may be present when patients live in different counties. The diagnosis must have been made between 1st April 1982 and 31st March 1984.

Individual hair dyes, along with their combinations, were tested for to see if they increased the chance of developing breast cancer. Here, there was no association between the use of hair dye, a diagnosis of BBD (at any age) and the development of breast cancer. In this study population, 889 women had BBD. If a woman had BBD but had never used hair dye the adjusted OR of her developing breast cancer was $1.67(95 \% \mathrm{CI}=1.28-2.20)$. If a woman had BBD and used hair dye, the adjusted OR of her developing breast cancer was $1.92(95 \% \mathrm{CI}=1.55-2.38)$. A clear overlap between the two confidence intervals can be seen here.
The ever use of hair dye correlated with the development of breast cancer with an OR of 1.04 (95\%CI=0.9-1.21).

Study 4: Hair dye use and breast cancer: a case-control study among screening participants (9). A standardised telephone interview was used to collect data in this retrospective case-control study conducted among patients from a screening centre in New York City. A total of 398 cases and 790 controls were used. Both groups were selected between 1977 and 1981. A complete history of hair dye use was taken, along with a range of confounding factors which were known or suspected influencers of the risk of developing breast cancer. Two factors decreased bias: interviewers were not informed whether the participant was a case or control and participants were told breast cancer risks were being investigated (hair dye was not specified at any point). Surrogate interviews, in cases of death/extreme illness, were conducted for 28 cases and 7 controls.

The study reports that the participants were "predominantly well educated, white, and largely of the Jewish religion, with a median age in years in the late 50's". The trend towards a specific subset of women indicates that these results must be treated with caution when applied to different socioeconomic or racial subgroups; they cannot generally be applied to all women.

There are no individual results for each kind of dye because some patients could not identify which type they had used (temporary, semi-permanent, and permanent). As individual data sets for types of hair dye are not provided, it is difficult to distinguish between dyes and discern a particularly harmful effect from any one of them. However, the 'ever' use of hair dye could be adequately assessed using the data provided. 
A key issue, raised within the study itself, is that of selection bias. Some women may not have received their cancer diagnosis through screening (for example if they were checked by a doctor when they became symptomatic), so they would not be included in this study. As screening is a voluntary effort there may be overrepresentation within this sample of certain types of women; women who attend screening appointments are likelier to be more educated and affluent. Age is another factor which may have had an effect, because screening is not recommended until women are over fifty years old. These various selection factors have been included in the multivariate logistic model and subgroups which are potentially biased were progressively removed from the analysis (for example women under 40 years of age). Despite the progressively homogenous nature of the analysis, the results remained similar.

The adjusted OR for a breast cancer patient having used hair dye was $0.8(95 \% \mathrm{CI}=0.6-1.1)$.

Study 5: Does hair dye use increase the risk of breast cancer? A population-based case-control study of Finnish women (10). A retrospective case-control study conducted in Finland, this population-based study used 6567 cases and 21598 controls between 22-60 years of age who underwent age matching. This is the largest study included in the meta-analysis. The use of the population-based cancer registry in Finland meant the selection bias was thought to be "negligible". A selfadministered quiz determined the use of hair dye amongst participants, examining: "cumulative number of hair dye episodes during life, age at first use and the types of dyes used". The age at which hair dye was first used was categorised: under 20 years, 20-29, 30-39, 40 years or older. The self-administered questionnaire method was examined by Shore et al., and was found to be valid. This is referenced within the study by Heikkinen et al. (10) The duration of hair dye from two telephone interviews one year apart with similar coefficients for cases and controls was similar $(r=0.86)(11)$. This potentially reduces the risk of recall bias for studies which did not conduct in-person interviews.

Multiple confounding factors were accounted for. If a patient used hair dye more than twice in their life, it is classified as the 'ever' use of hair dye. Zero, 1 or 2 applications of hair dye throughout the lifetime were classified as never use. The clear definition of 'ever' use of hair dye provides a standardisation tool when comparing the results of this epidemiological study to other studies. Participants who had deceased before the study had started were excluded. Although this means more aggressive cases may be missed, hair dye is such a widely used compound that this should not have a significant impact on the results, as clearly stated by the authors of this case-control study.

Hair dye use, in this study, is an indicator of a woman's use of other risk factors for breast cancer. For example, $7 \%$ of women who used hair dye did not drink as opposed to the $27 \%$ of women who did not use hair dye and did not drink. Risk factors were adjusted for. Socioeconomic status was considered the likeliest source of uncontrolled confounding bias.

The participants of this study were likely to be Caucasian, blonde and otherwise homogenous. As such, the results obtained should only be applied to populations with a Caucasian majority.

The biological basis of this epidemiological study is the use of 4-ABP, which was classified by the International Agency for Research on Cancer as carcinogenic for humans (16). There are concerns expressed in this paper that it is being used in hair dye products at concentrations above the legal limits, and it is suggested that certain potential carcinogenic effects of PPD are due to its contamination with 4-ABP during the production process. However, there is no focus on a specific chemical within this study; the focus was to determine if hair dye significantly increases the risk of breast cancer, and dyes are subdivided according to type (permanent, semi-permanent, temporary, bleach, partial) as opposed to specific chemicals found in them.

The OR for developing breast cancer following the use of hair dye was $1.23(95 \% \mathrm{CI}=1.11-1.36)$.

Study 6: Epidemiology of breast cancer and hair dyes (12). This is a retrospective case-control study conducted using 401 breast cancer patients and 625 controls who were white, 20-80 years of age and participants admitted to Memorial Sloan-Kettering Cancer Centre Between June 1979 and February 1981. The criteria for controls were as follows: they were admitted to the MSK Cancer Centre within two months of the breast cancer cases, they must not have a primary diagnosis of breast cancer, they must not have any medical history of breast cancer and they must not have had a diagnosis of BBD within a year of the study. The controls were matched by age at diagnosis within three years, as opposed to matched by age itself. The 'ever' use of hair dye is classified as "one or more applications of a particular product during the individual's lifetime".

Interviews were conducted via a trained interviewer in person administering a standardised questionnaire. The patients were shown images of 60 hair dye brands as memory aids and then asked to select what they had used. Family history and other potential confounding factors were examined: demographic data, family history, medication history, dietary history, occupational history, gynaecological history and use of known risk factors such as alcohol, tobacco, caffeine, and saccharin. Participants were stratified according to the risk factors.

Because all participants of this study were selected from a cancer centre, there is a high risk of selection bias; women who were treated here tended to be "well educated, in a high socioeconomic stratum, and Jewish". The greater number of Jewish women as cases (42.6\%) instead of controls (25.5\%) 
was significant $(p<0.001)$. The association of hair dye use with religion was significant $(p=0.003)$. For subsequent analyses, case-control comparisons were adjusted. However, these results may not be reflective of other populations.

Any women with diseases thought to be associated with the use of hair dye (apart from breast cancer) were excluded from the study. When the control group was dichotomised into cancer and non-cancer subgroups, "no significant main effects" were seen for any hair dye use associated variables. Wynder and Goodman assessed all the studies conducted prior to their own and discussed the other published results in tandem with the faults in these investigations.

The OR for developing breast cancer following the use of hair dye was 1.02 (95\% CI=0.77-1.32).

Study 7: Hair colouring, stress, and smoking increase the risk of breast cancer: A case-control study (13). This is a population-based study conducted in Iran which used 526 newly diagnosed cases and 526 controls frequency matched on age and chosen from Namazi Hospital in the period November 2014-March 2016. The cases were based on a pathology report and were undergoing radio/chemotherapy in the hospital at the time of the study. The controls were admitted during the same period due to non-neoplasm diseases and did not disclose any previous diagnosis of cancer. The focus on non-neoplasm diseases helps reduce any confounding effect which could have been introduced were any other diseases at admission included.

An "interview-administered questionnaire" was used to collect data. Confounding factors were tested for along with information regarding their use of hair dye. As there are multiple risk factors focussed on throughout the study, more detail regarding its use (type, longevity and duration of use, age at first use) was not achieved.

A multivariate analysis was used to determine if there was an increased risk of developing breast cancer following the use of hair dye. A menopause-stratified analysis was conducted to account for the biological and hormonal changes associated with this significant milestone. However, the results of the menopause and non-menopause participants were so similar that this data is not presented. The occurrence of breast cancer is recorded here as opposed to the survival of the patient following the diagnosis. As with any retrospective study, the risk of recall bias is present.

The author of this study underlines how the population of Iran is incredibly different to that of the West. He also points out how Iranian women have undergone huge epidemiological changes over the last few decades; for example, the birth rate dropped from over 7 children per woman to 1.7 over the course of approximately thirty years (early 1980s to 2007). Therefore, the epidemiological differences between Iranian and Western women are such that it would be difficult to apply these results to Western women.
The OR for developing breast cancer following the use of hair dye was $1.93(95 \% \mathrm{CI}=1.41-2.62)$. This study was the outlier of the funnel plot.

Study 8: Hair product use and the risk of breast cancer in young women (14). This retrospective case-control study was conducted across three counties of Washington using white female residents who fit the following criteria: 844 cases aged 45 or less and diagnosed with breast cancer in the period 1983-1990, and 960 controls of a similar age. Inperson interviews were utilised (excepting 32 participants who were interviewed over telephone) to ascertain information regarding participants' hair product use. The key focus of this investigation is hair dye; however, some attention is detracted from hair dye to hair spray.

The cases were identified using the Cancer Surveillance System in Western Washington. Cases had a "first invasive" breast cancer. Patients who had died prior to interview were excluded from the study. One patient had an unknown application of hair colouring and she was also excluded. Identification of controls occurred through random digit dialling. Controls were frequency age matched to cases within five years of themselves. Participants were given photographic displays and calendars which recorded major life events to prompt recall. Those interviewed by telephone were done so following receipt of the calendars/photographs by post. Women were questioned regarding their hair dye use, hair spray use and any exposure to known risk factors for cancer prior to their diagnosis (lifestyle and demographic, reproductive and substance use history).

Adjustments for the multivariate risk estimates were as follows: age, parity, weight, history of breast cancer in first degree relatives. Confounding risk factors were also adjusted for. Separate analyses were conducted for: women diagnosed with invasive and in situ breast cancer, hair colouring application before and after hair started turning grey, women with at least four instances of hair colour application. Similar results to the original set were produced for each, although they are not provided in the study.

Risk was assessed, and data provided for women with any use of hair dye, including non-exclusive use, and women with exclusive use of one type of hair dye. Women with any use of hair dye classify as the 'ever' use. The increase in breast cancer risk "appeared to be driven by" women using combinations of hair dye, not by exclusive use of a single type. This led the investigators to conclude that hair colouring application does not influence breast cancer risk.

The OR for developing breast cancer following the use of hair dye was $1.3(95 \% \mathrm{CI}=1.0-1.6)$.

Other systematic reviews/meta-analyses examining the relationship between the use of hair dye and the risk of developing breast cancer. We sourced two other meta- 
analyses which looked at whether there was an increased risk of developing human cancer after using hair dyes: Hair dye use and risk of human cancer and Personal Use of Hair Dyes and Risk of Cancer: A Meta-analysis.

Hair Dye use and Risk of Human Cancer (30). Eleven casecontrol and four cohort studies when examining the relationship between breast cancer and hair dye in this systematic review. The relationship between the incidence of different cancers and the use of hair dye was scrutinised. All studies included in this systematic review have also been cited in this meta-analysis (5-6, 8-9, 11-12, 14-18, 20-21, 23, $26,28)$. Exposure was only established through a single simple questionnaire at the start of the study. They highlighted some positive associations when the duration of use and type of hair dye use were considered in certain studies. A 2.5 -fold $(95 \% \mathrm{CI}=1.6,3.9)$ increased risk was found among women who reported using any hair dye products after bleaching (14). Within this study, there was also a range of other results, for example: a significantly increased risk was also observed for women who reported using any rinse $(\mathrm{OR}=1.7,95 \% \mathrm{CI}=1.2,2.5)$ and any frosting/tipping $(\mathrm{OR}=1.5,95 \% \mathrm{CI}=1.2,2.0)$ before applying hair dye products; and among women who reported using two or more types of hair dye products, a 3.1 -fold $(95 \% \mathrm{CI}=1.6$, 6.1) increased risk of breast cancer was observed for those who used hair dyes for 90 or more total episodes during their lifetime. Furthermore, for individuals using exclusively semipermanent types of hair colouring products, the OR was elevated (6). An increase in risk was also noted for women who changed hair colours multiple times (8). Two of the five cohort studies $(21,29)$ examined the mortality rate as opposed to the incidence of breast cancer, and this runs the risk of excluding many breast cancer cases which did not result in death. Both studies used the same cohort which was analysed at two different time points. These observations underscore the complexity of investigating the relationship between the personal use of hair dyes and breast cancer and emphasize the need for well-designed prospective population studies. The authors' suggestion that genetic polymorphism could modify the risk deserves further investigation, although this concept was applied in the systematic review specifically to the different aetiologies of the majority of Non-Hodgkin's Lymphoma diagnoses.

Personal Use of Hair Dyes and Risk of Cancer (31). Fourteen studies were used in this meta-analysis to examine the relationship between the use of hair dye and breast cancer to draw the overall conclusion that the "randomeffects pooled RR of breast cancer for any type of hair dye use was 1.06 (95\% CI, 0.95-1.18)". Twelve studies were case-control and 2 were cohort. All studies included by Takkouche et al. have been cited in our meta-analysis (5-6,
8-9, 11-12, 14-15, 17-18, 20, 23, 26, 29). The largest cohort study included in this meta-analysis (29) investigated the relationship between the personal use of hair dye and mortality from multiple cancers including that of the breast, which introduces the risk of underestimating the number of breast cancer events. This cohort study did not report any positive association between the personal use of hair dyes and breast cancer related mortality and this finding factored significantly in the meta-analysis due to its large sample size. The mortality data sets were obtained from cancer registries which are recognised to have a limited accuracy. This metaanalysis investigated multiple cancers hence breast cancer was not the sole focus of their investigation. There was a "moderate to large" degree of heterogeneity across all studies $(0.68)$, which could be explained by the inclusion of a casecontrol study which has a RR of 8.62 (20). Upon the exclusion of this study, the level of heterogeneity dropped down to 0.36 . Egger's test for the asymmetry present in the funnel plot yielded $p=0.90$ with the outlier and $p=0.72$ without.

Furthermore, the authors of this meta-analysis chose to include many studies which were excluded by us $(11,15,17$, $18,20,23,26,29)$. The study by Shore et al. (11) was relatively small and did not report the overall RR and $95 \%$ CI. The two studies which have been recorded and weighed separately by this meta-analysis $(15,18)$ are in fact using the same cohort. The different RRs appear because one is taken from the data set in Toronto and the other is taken from the data set in London. This small study was excluded by us because there was no average provided for the combination of the London and Toronto users and because in total, from Toronto and London, only 85 breast cancer patients were used. Takkouche et al. also chose to include the report by Petro-Nustas et al. (20) which has several limitations including a very small sample size and use of a convenience control group. Our strict inclusion criteria led to the exclusion of other studies which failed to adequately control for confounding factors $(23,26)$ or provide an accurate RR and $95 \%$ CI (17). We excluded 29 for reasons listed above.

Criteria for future studies concerning the relationship between breast cancer and hair dye. All chemical compounds used in hair dyes which are considered mutagenic and carcinogenic according to in vitro and in vivo animal studies must be identified at the outset and the presence of these components and their actual concentrations in the hair dye used are accurately recorded.

The ideal study to investigate this association would be a prospectively designed cohort study of a large sample size with accurate recording of background demographics that could affect the future risk of developing breast cancer. Assuming an annual incidence rate of 200 per 100,000 female participants aged 40 years or older and assuming a 
$35 \%$ exposure rate a sample size of $1,360,000$ would be needed to allow a robust statistical analysis with $80 \%$ power and 2-sided 5\% significance level to detect a RR of 1.1 (or 0.9 ) during a 5 year period of follow up. To demonstrate a RR of 1.2 or 0.8 (with $80 \%$ statistical power and a 2 -sided $5 \%$ significance level), the required sample size decreases to 356590 women (32).

The participants must not have any previous history of cancer, they must not be undergoing any radiotherapy or chemotherapy at the time of recruitment and they must not have genes predisposing them to be at a high risk of breast cancer (for example $B R C A 1$ and $B R C A 2$ ). The participants can be recruited from breast cancer screening centres that start screening mammography at the age of 40 and assessed for inclusion suitability through an initial questionnaire. The baseline demographics must include accurate recording of data regarding important risk factors for breast cancer such as family history, reproductive factors (parity and age at puberty), socioeconomic status, alcohol intake, use of hormone replacement therapy and the oral contraceptive pill, smoking habits, body mass index (BMI), previous breast biopsy, mammographic density and physical activity.

A standardised questionnaire should be prepared which is initially sent out to potential participants to determine suitability for inclusion. Then a detailed questionnaire is sent to selected participants to accurately determine the baseline characteristics listed above in addition to determining the extent of exposure to hair dyes including initial use, frequency and type of product and whether the use has changed over time. A follow-up questionnaire should be sent at 2-3 years after recruitment to ascertain any change in exposure characteristics. Ideally in person interviews should be conducted with visual aids as prompts. These interviews should be conducted at the beginning of the study, at regular intervals throughout the study and at the end of the study. Outcomes are recorded as lack/development of breast cancer as the primary endpoint; mortality does not need to be examined. It is expected that within 5 years of completing recruitment the study will have sufficient statistical power to answer questions related to the potential impact of personal use of hair dyes on breast cancer risk. Prospective cohort studies are expensive, time-consuming and vulnerable to attrition bias. The multibillion dollar hair dyes industry could be invited to participate in the funding of such studies.

A large retrospective case-control study with individual matching and the use of multiple controls with measures to minimise the risk of recall and interviewer bias and ensure accuracy of data recording would represent a faster and cheaper alternative to prospective cohort studies. The selection criteria for cases and controls must be carefully established and baseline category information must be accurately recorded. These potential confounding factors must be stratified or accounted for in a multivariate analysis (33).

\section{Conclusion}

Our study suggests that the personal use of hair dyes may be associated with an increased risk of breast cancer. Consumers should be aware of this potential association; however, to make informed choices, further research is required to confirm our results and clarify the possible mechanisms using the criteria we have outlined above. Based on the current knowledge, it is reasonable that cosmetic industry regulators consider limiting the concentration of the potentially carcinogenic chemical PPD to a maximum $2 \%$ as recommended by the Scientific Committee on Consumer Safety of the European Commission which could not draw conclusions regarding its carcinogenicity in humans (34) and manufacturers should adhere to such guidelines and accurately label hair products. The European Union Cosmetic Directive currently allows a maximum PPD concentration of up to $6 \%$ in hair dyes in the context of allergy (35). Our findings do not represent evidence for the presence of a cause-effect relationship.

\section{Acknowledgements}

We are very grateful to Breast Cancer Hope Charity for supporting our research.

\section{References}

1 Hair Dyes and Cancer Risk [Internet]. National Cancer Institute. 2016 [cited 12 September 2017]. Available from: https:// www.cancer.gov/about-cancer/causes-prevention/risk/myths/hairdyes-fact-sheet\#r1

2 List of 181 substances banned for use in hair dye products [Internet]. European Commission, growth: internal market, industry, entrepreneurship and SMEs. 2015 [cited 22 September 2017]. Available from: http://ec.europa.eu/DocsRoom/ documents/13209/attachments/1/translations

3 Chong HP, Reena K, Ng KY, Koh RY, Ng CH and Chye SM: Para-phenylenediamine containing hair dye: An overview of mutagenicity, carcinogenicity and toxicity. J Environ Anal Toxicol 6(5): 403, 2016.

4 IARC Working Group on the Evaluation of Carcinogenic Risk to Humans: Some aromatic amines, organic dyes, and related exposures. In: Iarc monographs on the evaluation of carcinogenic risks to humans. IARC Monographs, IARC Vol 99, 2012.

5 Nasca PC, Lawrence CE, Greenwald P, Chorost S, Arbuckle JT and Paulson A: Relationship of hair dye use, benign breast disease, and breast cancer. J Natl Cancer Inst 64(1): 23-28, 1980.

6 Zheng T, Holford T, Mayne S, Owens P, Boyle P, Zhang B, Zhang $\mathrm{Y}$ and Zahm S: Use of hair colouring products and breast cancer risk: A case-control study in connecticut. Eur J Cancer 38(12): 1647-1652, 2002.

7 Pressler M, Rosenberg CA, Derman BA, Greenland P, Khandekar J, Rodabough RJ, McTiernan A and Simon MS: Breast cancer in postmenopausal women after non-melanomatous skin cancer: The women's health initiative observational study. Breast Cancer Res Treat 139(3): 821-831, 2013. 
8 Nasca PC, Baptiste MS, Field NA, Metzger BB and DeMartino $\mathrm{R}$ : An epidemiologic case-control study of breast cancer and exposure to hair dyes. Ann Epidemiol 2(5): 577-586, 1992.

9 Koenig KL, Pasternack BS, Shore RE and Strax P: Hair dye use and breast cancer: A case-control study among screening participants. Am J Epidemiol 133(10): 985-995, 1991.

10 Heikkinen S, Pitkäniemi J, Sarkeala T, Malila N and Koskenvuo M: Does hair dye use increase the risk of breast cancer? A population-based case-control study of finnish women. PloS one 10(8): e0135190, 2015.

11 Shore RE, Pasternack BS, Thiessen EU, Sadow M, Forbes R and Albert RE: A case-control study of hair dye use and breast cancer 2. J Natl Cancer Inst 62(2): 277-283, 1979.

12 Wynder EL and Goodman M: Epidemiology of breast cancer and hair dyes 2. J Natl Cancer Inst 71(3): 481-488, 1983.

13 Dianatinasab M, Fararouei M, Mohammadianpanah M, Zarebandamiri $\mathrm{M}$ and Rezaianzadeh A: Hair coloring, stress, and smoking increase the risk of breast cancer: A case-control study. Clinical Breast Cancer pii: S1526-8209(17)30135-0, 2017.

14 Cook LS, Malone KE, Daling JR, Voigt LF and Weiss NS: Hair product use and the risk of breast cancer in young women. Cancer Causes Control 10(6): 551-559, 1999.

15 Stavraky KM, Clarke EA and Donner A: Case-control study of hair dye use by patients with breast cancer and endometrial cancer 2. J Natl Cancer Inst 63(4): 941-945, 1979.

16 Hennekens C, Speizer F, Rosner B, Bain C, Belanger C and Peto $\mathrm{R}$ : Use of permanent hair dyes and cancer among registered nurses. The Lancet 313(8131): 1390-1393, 1979.

17 Kinlen L, Harris R, Garrod A and Rodriguez K: Use of hair dyes by patients with breast cancer: A case-control study. Br Med J 2(6083): 366-368, 1977.

18 Stavraky KM, Clarke EA and Donner A: A case-control study of hair-dye use and cancers of various sites. Br J Cancer 43(2): 236-239, 1981.

19 Llanos AA, Rabkin A, Bandera EV, Zirpoli G, Gonzalez BD, Xing CY, Qin B, Lin Y, Hong C-C and Demissie K: Hair product use and breast cancer risk among african american and white women. Carcinogenesis 38(9): 883-892, 2017.

20 Petro-Nustas W, Norton ME and Al-Masarweh I: Risk factors for breast cancer in jordanian women. J Nurs Scholarsh 34(1): 19-25, 2002.

21 Thun MJ, Altekruse SF, Namboodiri MM, Calle EE, Myers DG and Heath CW Jr.: Hair dye use and risk of fatal cancers in us women. JNCI 86(3): 210-215, 1994.

22 Skov T and Lynge E: Cancer risk and exposures to carcinogens in hairdressers. Skin Pharmacol 7(1-2): 94-100, 1994.
23 Green A, Willett WC, Colditz GA, Stampfer MJ, Bain C, Rosner B, Hennekens $\mathrm{CH}$ and Speizer FE: Use of permanent hair dyes and risk of breast cancer 2. J Natl Cancer Inst 79(2): 253-257, 1987.

24 Hartz AJ and He T: Cohort study of risk factors for breast cancer in post menopausal women. Epidemiol Health 35: e2013003, 2013.

25 Pollán M and Gustavsson P: High-risk occupations for breast cancer in the swedish female working population. Am J Public Health 89(6): 875-881, 1999.

26 Boice JD, Mandel JS and Doody MM: Breast cancer among radiologic technologists. JAMA 274(5): 394-401, 1995.

27 Vedel-Krogh S, Nielsen SF, Schnohr P and Nordestgaard BG: Morbidity and mortality in 7,684 women according to personal hair dye use: The copenhagen city heart study followed for 37 years. PloS One 11(3): e0151636, 2016.

28 Mendelsohn JB, Li QZ, Ji BT, Shu XO, Yang G, Li HL, Lee $\mathrm{KM}, \mathrm{Yu} \mathrm{K}$, Rothman N and Gao YT: Personal use of hair dye and cancer risk in a prospective cohort of chinese women. Cancer Sci 100(6): 1088-1091, 2009.

29 Altekruse SF, Henley SJ and Thun MJ: Deaths from hematopoietic and other cancers in relation to permanent hair dye use in a large prospective study (united states). Cancer Causes Control 10(6): 617-625, 1999.

30 Zhang Y, Kim C and Zheng T: Hair dye use and risk of human cancer. Front Biosci (Elite edition) 4: 516, 2012.

31 Takkouche B, Etminan M and Montes-Martínez A: Personal use of hair dyes and risk of cancer: A meta-analysis. JAMA 293(20): 2516-2525, 2005.

32 Lwanga SK, Lemeshow S and Organization WH: Sample size determination in health studies: A practical manual, Geneva: World Health Organization, 1991.

33 Risk factorslBreast cancerlCancer Research UK [Internet]. Cancerresearchuk.org. 2017 [cited 29 September 2017]. Available from: http://www.cancerresearchuk.org/about-cancer/ breast-cancer/risks-causes/risk-factors

34 European Commission, Scientific Committee on Consumer Safety. Opinion on p-Phenylenediamine, 17-18 September, 2012.

35 Kim K-H, Kabir E and Jahan SA: The use of personal hair dye and its implications for human health. Environ Int 89: 222-227, 2016.
Received October 6, 2017

Revised November 29, 2017

Accepted December 18, 2017 